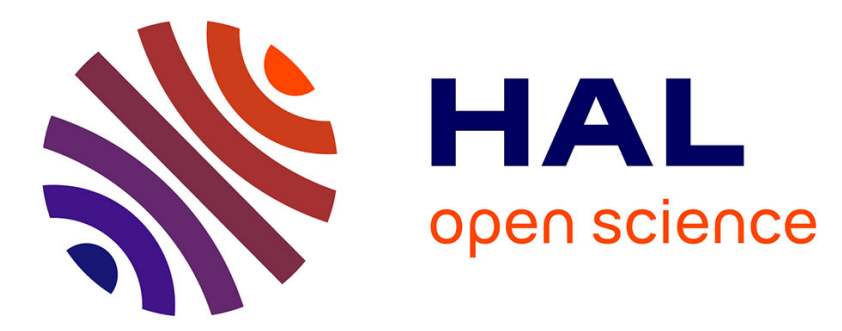

\title{
Vulnerability Analysis of Critical Infrastructure under Disruptions: an Application to China Railway High-Speed Acknowledgement
}

Chao Fang, Piao Dong, Yiping Fang, Enrico Zio

\section{- To cite this version:}

Chao Fang, Piao Dong, Yiping Fang, Enrico Zio. Vulnerability Analysis of Critical Infrastructure under Disruptions: an Application to China Railway High-Speed Acknowledgement. Proceedings of the Institution of Mechanical Engineers, Part O: Journal of Risk and Reliability, inPress, 10.1177/1748006X19889149 . hal-02431422

\section{HAL Id: hal-02431422 \\ https://hal.science/hal-02431422}

Submitted on 7 Jan 2020

HAL is a multi-disciplinary open access archive for the deposit and dissemination of scientific research documents, whether they are published or not. The documents may come from teaching and research institutions in France or abroad, or from public or private research centers.
L'archive ouverte pluridisciplinaire HAL, est destinée au dépôt et à la diffusion de documents scientifiques de niveau recherche, publiés ou non, émanant des établissements d'enseignement et de recherche français ou étrangers, des laboratoires publics ou privés. 


\title{
Vulnerability Analysis of Critical Infrastructure under Disruptions: an Application to China Railway High-Speed
}

\author{
Chao FANG1, Piao DONG ${ }^{2}$, Yiping FANG ${ }^{3 *}$, Enrico ZIO ${ }^{4,5}$

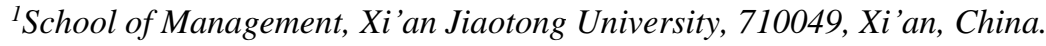 \\ ${ }^{2}$ Economics and Management School, Wuhan University, 430072, Wuhan, China. \\ ${ }^{3}$ Chaire Systems Science and the Energy Challenge, Fondation Electricité de France (EDF), \\ Laboratoire Génie Industriel, CentraleSupélec, Université Paris-Saclay, 3 Rue Joliot Curie, 91190 \\ Gif-sur-Yvette, France. \\ ${ }^{4}$ Mines ParisTech, PSL Research University, CRC, Sophia Antipolis, France. \\ ${ }^{5}$ Energy Department, Politecnico di Milano, Via La Masa 34, Milano, 20156, Italy.
}

*Corresponding author: Yiping FANG

Email address: yiping.fang@centralesupelec.fr

\section{Acknowledgement}

The authors sincerely thank the editors and the anonymous reviewers for their invaluable comments and suggestions for improving this article. This work was supported in part by the NSFC under Grant 71731008, and by "Academic Team Building Plan for Young Scholars from Wuhan University”. 


\begin{abstract}
Considerable attention has been paid to the vulnerability of critical infrastructures, because of the increasing occurrence of disruptive events, such as man-made or natural disasters. Even small disruptions could eventually affect the normal function of infrastructure systems. Enhancing the reliability of these systems and their robustness to disruptions is necessary and urgent. High-speed rail is a critical infrastructure that is subject to various disruptions, including component aging, malicious attacks, natural disasters, demand surges. In this study, we analyze the topological centrality indicators of China Railway High-speed (CRH) network using network theory, and take real train flow information for assessing the importance of network components in terms of vulnerability to disruption. By Monte Carlo simulation, we analyze the risk of the CRH network under random attacks and spatially localized failures. The significance of taking pre-actions for protecting critical infrastructures by mitigating its vulnerability to disruptions is emphasized.
\end{abstract}

Keywords: vulnerability analysis; disruption; critical infrastructures; Monte Carlo simulation; spatially localized failures

\title{
1. Introduction
}

Critical infrastructures are large dynamic systems made of interconnected components and sub-systems. They provide products and services, such as transportation, energy, communication, water supply, finance, and public health. Such critical infrastructures are vital to the normal functioning of society, and their failure can seriously affect public safety, economic development and social stability ${ }^{1}$. In recent years, critical infrastructures are facing increasing risks and security threats, e.g., operation failures, natural disaster, and terrorist attacks. Examples include the Deepwater Horizon oil spill, the accident at Fukushima nuclear power plant, the Haiti earthquake, and the Puerto Rico hurricane ${ }^{2}$. The vulnerability analysis of critical infrastructures has recently become of particular interest and many studies have been conducted on this subject. For example, some researchers ${ }^{3-6}$ discussed the protection of critical infrastructures and related challenges, of which Marrone et al. 5 introduced a systemic view for the practical protection of critical infrastructures and demonstrated its effectiveness with an application to industrial settings. From the perspective of methodologies and applications, Zio ${ }^{7}$ presented a vulnerability framework and indicated the importance of integrating different modeling approaches facing the dynamic complexities of critical infrastructure. Johansson et al. ${ }^{8}$ and Ouyang ${ }^{9}$ offered technical reviews of approaches for the reliability and vulnerability analysis of critical infrastructures. 
Transport infrastructures, usually distributed on geographical extensions, are collections of many interacting man-made components assembled by design, for moving goods and people from one place to another. It can be termed critical infrastructure if its incapacity has a significant impact on health, safety, security, economics and social well-being ${ }^{7}$. The growing vulnerabilities of transportation systems force decision makers to prioritize their protection. In this respect, some studies ${ }^{10-12}$ discussed the vulnerability analysis of road transportation systems with indicators used in the modeling that can be roughly divided into demand and supply. By using exhaustive search optimization and fuzzy logic, El-Rashidy and Grant-Muller ${ }^{13}$ presented a model that estimated the vulnerability changes of the highway network under different situations. $\mathrm{Li}$ and $\mathrm{Cai}{ }^{14}$ focused on the Chinese railway weighted network and found that the network has small-world and scale-free characteristics. Johansson and Hassel ${ }^{15}$ modeled an electrified railway network, explored its five subsystems and analyzed the interdependencies among them for vulnerability assessment. Ouyang et al. ${ }^{16}$ systematically analyzed the positive effect of interdependencies in complementary systems and proposed a network-based approach to model the vulnerability of transportation systems. Bocchini et al. ${ }^{17}$ proposed a comprehensive method for assessing the vulnerability of a railway system under floods, and compared four vulnerability mitigation and maintenance strategies.

In general, a large number of the existing studies on vulnerability and reliability analysis of railway transportation systems have focused on rescheduling or reassignment of train services, following disruptions ${ }^{18-22}$. In terms of economic efficiency and social function, preparedness to and mitigation of the disruption risks to decrease vulnerability is also considerably important. Furthermore, the performance metrics used in existing research are often topological, with only few exceptions considering railway flow ${ }^{23-26}$. However, these studies usually refer to the number of train service lines affected as system performance indicator, which is insufficient because the importance of each service line is not the same, especially for passenger trains.

High-speed rail is an important part of a rail transportation system, the development of which has become a global trend since 1964 when the world's first high-speed railway, Japan's Shinkansen, opened to traffic. Regional integration, urban and regional agglomeration, and increasing labor productivity are key factors that push the emergence of high-speed rail worldwide ${ }^{27}$. Given its characteristics of punctuality, comfort, energy efficiency and reduced pollution, high-speed rail has obtained wide support, leading to "the second railway age" 28 . With the importance and influence of high-speed rail worldwide, the ability to predict, withstand, and mitigate disruptions has raised considerable attention from managers and researchers. Given that high-speed rail provides services for mass passengers, the loss caused by a disruption is considerably more serious than that of freight trains. High-speed rail is also 
more sensitive to time, given the competition from civil aviation. The high-speed rail system is a crucial yet sensitive infrastructure of a nation's transportation systems and even a small disruption may lead to significant adverse effects not only on the economy but also on public safety.

Official data indicates that the railway mileage of China reached $124,000 \mathrm{~km}$ by 2017 , of which high-speed rail operating mileage exceeded 22,000 km, accounting for more than $60 \%$ of the world's high-speed rail ${ }^{29}$. From a practical perspective, some researchers ${ }^{28,} 30-32$ considered the characteristics and future development of the China Railway High-speed (officially named CRH). However, the analysis of CRH's vulnerability with quantitative metrics has yet to be conducted. In the present study, vulnerability is considered as decline in system performance. We analyze not only the system vulnerability of CRH using topological centrality indicators, but also consider the train flow information in operation to assess the importance of network components in terms of vulnerability to disruption. To indicate the difference in the importance of different trips, the mileage of train service line in operation is used in our flow-based vulnerability analysis. The application to the CRH network shows how these analyses can provide complementary information and knowledge of the vulnerability caused by disruptions and dynamic flows in railway systems. We use the Monte Carlo method and spatially localized failures (SLFs) ${ }^{33,34}$ to simulate disruptions, like random attacks and natural disasters. The insights from this study can assist local and central government agencies in making decisions to enhance and protect $\mathrm{CRH}$ networks with respect to vulnerability mitigation.

The remainder of this paper is organized as follows. In Section 2, we introduce a network modeling of $\mathrm{CRH}$, in which its topological characteristics are analyzed to gain a general structural understanding with regard to vulnerability. Section 3 presents a vulnerability analysis considering dynamic flows in the network. In Section 4, we simulate spatially localized failures using the Monte Carlo method and illustrate the vulnerability intensity chart of the CRH. Further managerial discussion is given in Section 5 and conclusions are drawn in Section 6.

\section{Notations}

$N: \quad$ set of nodes (railway stations), $n_{i} \in N$

$L: \quad$ set of links (railway links connecting stations), $l_{i} \in L$

$G(N, L)$ : railway network comprising a set of nodes $N$ connected by a set of links $L$

$g_{i j}: \quad$ actual geodesic distance between $n_{i}$ and $n_{j}$

$l_{i j}$ : link connecting two adjacent nodes $i$ and $j$

$n_{u v}: \quad$ number of shortest paths that connect $n_{u}$ and $n_{v}$

$n_{u v}(i)$ : number of shortest paths connecting $n_{u}$ and $n_{v}$, as well as passing through $n_{i}$ 
$n_{u v}(i j)$ : number of shortest paths connecting $n_{u}$ and $n_{v}$, as well as passing through $l_{i j}$

$T: \quad$ total number of all train services, $t \in T$

TTM: $\quad$ total travel mileage of all train services within the network

CTM $_{i}$ : $\quad$ cumulative travel mileage affected by failure of $n_{i}$

$C T M_{i j}: \quad$ cumulative travel mileage affected by failure of $l_{i j}$

$A T M_{i}^{t}$ : adjacent travel mileage of the train that passes $n_{i}$

$T M_{i}^{t}: \quad$ travel mileage of the train that starts/ends from $n_{i}$

$T M_{i j}^{t}$ : travel mileage of the train that includes $l_{i j}$

\section{Network modeling and topological analysis of CRH}

\subsection{Network modeling}

From the perspective of complex network theory, an infrastructure can be described as a network graph $G(N, L)$, where $N$ represents the set of components (nodes) of the system and $L$ the set of connections (lines) between the components. Given the topology of the infrastructure system, represented by a graph-based model, we can analyze its structural properties with respect to suffered disruptions.

On August 1, 2008, CRH began its debut operation. As of January 2017, the main skeleton of CRH's "four vertical and four horizontal" structure has been completed. This structure plays an important role in interregional passengers' transport in China. The infrastructure has been significantly developed and nowadays CRH is leading the development of high-speed railways worldwide. At present, CRH is operational in 296 cities, including 137 prefecture-level cities (municipalities, provincial cities and prefecture-level cities) and 159 county-level cities (in addition to counties outside the municipal districts). The CRH can be represented by a network $G(N, L)$, where $N$ is the set of stations and $L$ is the set of rail links connecting the stations. In this study, we assume that multiple stations in the same city are combined into one site. For example, the city of Wuhan houses three railway stations, namely, Wuhan, Wuchang, and Hankou Stations, and we abstract them into one site called Wuhan Station. Although numerous small sites are not included in the nodes set, their properties are accounted for in the links in the network. Finally, based on the CRH operation map ${ }^{35}$, we abstract it into a relatively simplified network diagram (see Figure 1), which contains 28 nodes (stations) and 42 links (railway tracks). The text in the Figure represents the city name, such as BJ for Beijing and SH for Shanghai. 


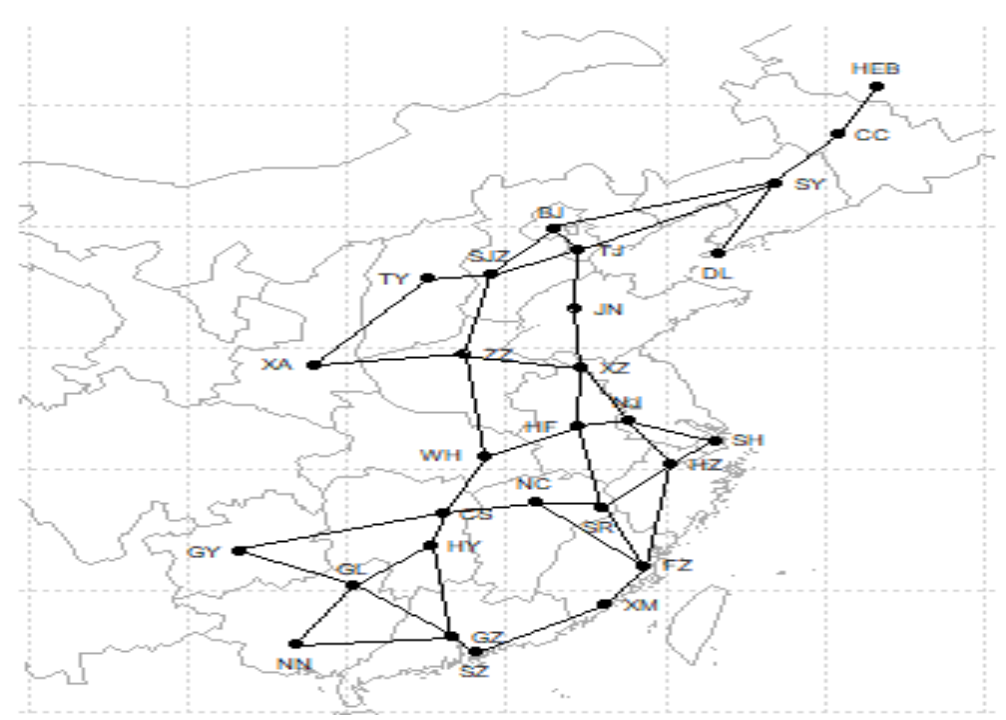

Figure 1. Simplified network representation of CRH

\subsection{Topological analysis}

Numerous studies have been conducted on the complexity characteristics and vulnerability of railway transport systems. These studies found that the vulnerability with regard to many types of disruptions in the system are closely related to the structural properties of the networks ${ }^{17,36,37}$. In this regard, we tailor some network indicators of betweenness centrality to characterize the topological properties of the $\mathrm{CRH}$ network with respect to vulnerability.

Betweenness includes node and link betweenness. It reflects the global connection importance of the components in the entire network. Here we consider the betweenness centrality based on shortest paths because in practice, the network design and route planning usually consider the shortest path as an important reference. The value of node betweenness is determined by the proportion of the number of paths that pass through this node in all the shortest paths. By defining $n_{u v}$ as the number of shortest paths that connect $n_{u}$ and $n_{v}$, $n_{u v}(i)$ as the number of shortest paths connecting $n_{u}$ and $n_{v}$ as well as passing through $n_{i}$, the betweenness of $n_{i}$ is calculated by:

$$
B_{i}=\sum_{u, v \in N, u \neq v} \frac{n_{u v}(i)}{n_{u v}}
$$

The calculation of a link betweenness is similar, where $n_{u v}(i j)$ represents the number of shortest paths connecting $n_{u}$ and $n_{v}$, as well as passing through $l_{i j}$ :

$$
B_{i j}=\sum_{u, v \in N, u \neq v} \frac{n_{u v}(i j)}{n_{u v}}
$$

Table 1 shows the ranking of the top-ten nodes and links based on betweenness centrality. The first three columns indicate that Zhengzhou, Wuhan, and Changsha Stations are the top three nodes that possess the highest betweenness. These nodes have important topological positions and include many paths passing by. Figure 1 shows that these stations are central hubs in the network running north and south. For example, although the degree of Wuhan 
Station node is 3 , it remains necessary for the connection of the north and south, due to its location in the central area of the network. Accordingly, the hub stations with high betweenness centrality are usually more sensitive to disruptions and once breakdown occurs, the entire network can be greatly affected. Table 1 shows also the top ten links with highest betweenness. The railway track from Wuhan to Changsha ranks premier. This is consistent with the results of node's betweenness, because these two nodes are in the central area of the network and each node has considerable betweenness value. These nodes and links are important in terms of topology and vulnerability, and hence mitigation and preparedness strategies should be considered in strengthening the components to resist potential disruptions.

TABLE 1. TOP 10 IMPORTANT NODES AND LINKS IN TERMS OF BETWEENNESS CENTRALITY

\begin{tabular}{ccccc}
\hline \multirow{2}{*}{ Ranking } & Nodes & $\mathbf{B}_{\boldsymbol{i}}$ & Links & $\mathbf{B}_{\boldsymbol{i j}}$ \\
\cline { 2 - 5 } 1 & Zhengzhou (ZZ) & 0.37681 & Wuhan-Changsha(WH-CS) & 0.28157 \\
2 & Wuhan (WH) & 0.36025 & Wuhan-Zhengzhou(WH-ZZ) & 0.26501 \\
3 & Changsha(CS) & 0.36025 & Shijiazhuang-Zhengzhou(SJZ-ZZ) & 0.25879 \\
4 & Shijiazhuang (SJZ) & 0.29193 & Hangzhou-Nanjing(HZ-NJ) & 0.14492 \\
5 & Guangzhou(GZ) & 0.21946 & Tianjin-Jinan(TJ-JN) & 0.12836 \\
6 & Nanjing(NJ) & 0.21532 & Jinan-Nanjing(JN-NJ) & 0.12629 \\
7 & Hangzhou (HZ) & 0.21118 & Changsha-Guangzhou(CS-GZ) & 0.11801 \\
8 & Jinan(JN) & 0.20911 & Xuzhou-Hefei (XZ-HF) & 0.10766 \\
9 & Shenyang(SY) & 0.20704 & Wuhan-Hefei(WH-HF) & 0.09937 \\
10 & Hefei (HF) & 0.20083 & Zhengzhou-Xuzhou(ZZ-XZ) & 0.09109 \\
\hline
\end{tabular}

\section{Flow-based vulnerability analysis under disruptions}

The impact caused by a disruption is mainly in the failure of services provided by the infrastructure, thereby causing inconvenience to people's lives and losses of finance. Different critical infrastructure systems have different physical flow characteristics of operation, such as electric flow in power systems ${ }^{38,39}$, train services in railway transportation systems, and information flow in telecommunication systems. With regard to $\mathrm{CRH}$, this study considers the flow of trains. Several train service lines are run back and forth to ensure people's travel, with duration of round-trips often less than 24 hours thanks to the high-speed. We have obtained the information of all train services from the website of China Railway Customer Service Center ("12306.cn"). We have selected all the high-speed trains that contain the prefix "G" for "GaoTie" (high-speed rail) and obtain 2,324 CRH train service lines in operation in a normal weekday. Given that the operating mileage of each train service line is not the same, its importance cannot be generalized when analyzing network vulnerability. Therefore, the train 
flow in this study takes into account the mileage of each train services, rather than simply the number of train service lines in operation.

By removing components in sequence from the network, we study the importance of each component separately. The vulnerability is defined here as the drop in system performance: thus, the importance of a component is measured by the effect on the proportion of the mileages of train service affected by the component's failure (removal). Most often, the current duration of a disruption in CRH is no more than one day. Thus, we assume that the failure of the components can be recovered within 24 hours and network congestion is not taken into account here. Furthermore, some other assumptions simplify the calculation, such as the facility failures being binary (completely operational or completely failed).

From the topological analysis of Section 2.2, we gain an interpretation of the components' importance in CRH. To learn more about the vulnerability of the infrastructure network, we introduce performance metrics related to the flow of train services. Here we use the performance metric of the network connectivity efficiency. Connectivity efficiency is calculated by the average geodesic distance $\left(g_{i j}\right)$ between two nodes belonging to the network. The distance is geodesic, which we consider equal to the railway track length determined by the train services between two components. Thus, this metric can reflect the connectivity efficiency of the network from a realistic point of view. The equation to calculate connectivity efficiency is:

$$
E=\frac{1}{N(N-1)} \sum_{i \neq j} \frac{1}{g_{i j}} .
$$

We indicate the connectivity efficiency of the network in the normal state as $E$. If a disruption on $n_{i}$ occurs, which invalidates $n_{i}$, then $E_{i}^{\prime}$ should be defined by the network efficiency excluding $n_{i}$. Therefore, the efficiency-based vulnerability under the event is calculated as follows:

$$
V_{i}^{E}=\frac{E-E_{i}^{\prime}}{E} .
$$

On the basis of the values of $V_{i}^{E}$, the ten most important railway stations of CRH are listed in Table 2. From the Table, Nanjing is the most important station in terms of connectivity efficiency. If a disruption occurs and shuts down Nanjing Station, then the connectivity efficiency of the network is reduced by $12.4 \%$. The next important two stations after Nanjing are Tianjin $\left(V_{\mathrm{TJ}}^{E}=0.116\right)$ and Hangzhou $\left(V_{\mathrm{HZ}}^{E}=0.110\right)$ stations. In CRH map we found that several nodes are connected to them and the geodesic distance is relatively small. For example, the geodesic distance between Nanjing and Hefei is only $175 \mathrm{~km}$ and that between Nanjing and Hangzhou is $276 \mathrm{~km}$, whereas the geodesic distances between other nodes are often more than $300 \mathrm{~km}$. The efficiency of the network is mainly determined by the geodesic distance. Moreover, if one of two close nodes fail, then the other one can only be 
reached through other remote nodes in the network, which may lead to a notable reduction in network efficiency. Therefore, these nodes are rather important in terms of network efficiency and should be given sufficient attention and protection.

TABLE 2. TOP 10 IMPORTANT NODES IN TERMS OF NETWORK EFFICIENCY VULNERABILITY

\begin{tabular}{ccc}
\hline \multirow{2}{*}{ Ranking } & Nodes & $\boldsymbol{V}_{\boldsymbol{i}}^{\boldsymbol{E}}$ \\
\cline { 2 - 3 } 1 & Nanjing(NJ) & 0.12366 \\
2 & Tianjin(TJ) & 0.11568 \\
3 & Hangzhou(HZ) & 0.11028 \\
4 & Shijiazhuang(SJZ) & 0.10681 \\
5 & Hefei(HF) & 0.10463 \\
6 & Guangzhou(GZ) & 0.10429 \\
7 & Changsha(CS) & 0.09611 \\
8 & Xuzhou(XZ) & 0.09415 \\
9 & Beijing(BJ) & 0.09288 \\
10 & Shangrao(SR) & 0.08947 \\
\hline
\end{tabular}

We also model the vulnerability regarding individual components from the perspective of affected operating mileage. If we assume a disruption on one node $i$, then the effect caused by the failure of this node should be quantified by the affected trains, as well as the affected cumulative travel mileages, denoted by $C T M_{i}$. TTM indicates the total travel mileage of all train services within network. Accordingly, the vulnerability of node $i$ considering train flow can be expressed as follows:

$$
V_{i}^{F}=\frac{C T M_{i}}{T T M} .
$$

From the practical point of view, we assume that if the start/end station of a train service line is interrupted, then the entire line is affected. That is the common situation because the train cannot be prepared to depart normally or reach its destination for replenishment and maintenance for the return trip. Meanwhile, by field investigation and interviewing experts in $\mathrm{CRH}$, we acknowledge that the interruption of intermediate stations along the line can possibly not affect the operation of the whole train service. That is because the train can choose not to stop at the disrupted station while moving on to the nearby dockable yard, or running across station in emergencies (especially for large stations having many linking routes). The train also has the option of returning in the network to ensure only a part of the original travel service.

Based on these assumptions, in this study, train services affected by the failures of stations can be divided into two types. When the start/end station $n_{i}$ of the service line is disrupted, the impact caused by this failure of $n_{i}$ should include the total travel mileages of these trains, as denoted by $T M_{i}^{t}$. The other situation is that the failure node is neither the starting point nor the terminal station of the train, where the impact is defined in our study by the travel mileages between this node and the adjacent stations of the affected trains, denoted 
by $A T M_{i}^{t}$. Denote $T$ as the total number of all trains, then we can calculate $C T M_{i}$ by:

$$
\left.C T M_{i}=\sum_{t=1}^{T} T M_{i}^{t} \text { (where } n_{i} \text { is start/end node for train } t\right)+\sum_{t=1}^{T} A T M_{i}^{t} \text { (otherwise) }
$$

For the case of link failure, as a link has absorbed a number of small yards not shown in the network structure, we assume that if the link between two adjacent stations is disrupted, then any train passing through it will be affected. Thus, the vulnerability of $l_{i j}$ should be quantified by the affected travel mileages for the proportion of the total travel mileages.

$$
V_{i j}^{F}=\frac{C T M_{i j}}{T T M}
$$

As illustrated in Table 3, the top-ten vulnerable stations are different from the previous topological rankings in Tables 1 and 2. The first three columns of Table 3 indicate that Shanghai, Beijing, and Guangzhou Stations are the three most important stations. This can be easily explained by the fact that Beijing is the junction of the Beijing-Shanghai, KazakhstanBeijing, Beijing-Hong Kong, and the Jingkun channels. Meanwhile, Shanghai is the junction of Beijing-Shanghai channel, the coastal channel, and the channel along the Yangtze River. Guangzhou has become a three main line intersection hub of the Kazakhstan-Beijing, LanGuang, and Guang-Kun channels. Once a disruption occurs in any of these three busiest stations, a greater effect will be incurred on the entire CRH system. The link failure analysis results reveal that the links connecting adjacent important nodes are also highly important in terms of vulnerability taking into account train flow, such as Shanghai--Nanjing track. In addition, some hub links with high topological betweenness centrality are also vulnerable, such as Wuhan-Changsha, Wuhan-Zhengzhou, that bear a lot of traffic flow in CRH.

Figure 2 is a visual representation of the results with the geographical location. We highlighted in the map the top-five nodes and top-ten links, whose failure can seriously affect the CRH operation flow. We can see that most top vulnerable nodes are connected by the top important links. Beijing, the capital, is ranked No.2 vulnerable station because of its numerous departure/terminal train services; however, the nodes and links connecting to Beijing are not ranked as high as expected because of its Siphon Effect: that is to say, the neighbor city stations around Beijing have been weakened in terms of their capacity of train flow in practice. 


\begin{tabular}{ccccc}
\hline \multirow{2}{*}{ Ranking } & Nodes & $\boldsymbol{V}_{\boldsymbol{i}}^{\boldsymbol{F}}$ & Links & $\boldsymbol{V}_{\boldsymbol{i} \boldsymbol{j}}$ \\
\cline { 2 - 5 } 1 & Shanghai(SS) & 0.28073 & Shanghai-Nanjing(SS-NJ) & 0.23488 \\
2 & Beijing(BJ) & 0.23519 & Wuhan-Changsha(WH-CS) & 0.18932 \\
3 & Guangzhou(GZ) & 0.13641 & Jinan-Xuzhou(JN-XZ) & 0.17250 \\
4 & Nanjing (NJ) & 0.13358 & Wuhan-Zhengzhou(WH-ZZ) & 0.16931 \\
5 & Wuhan(WH) & 0.13232 & Changsha-Hengyang(CS-HY) & 0.14632 \\
6 & Changsha(CS) & 0.11551 & Shijiazhuang-Zhengzhou(SJZ-ZZ) & 0.14437 \\
7 & Xian(XA) & 0.11196 & Nanjing-Hangzhou(NJ-HZ) & 0.13359 \\
8 & Zhengzhou(ZZ) & 0.09776 & Guangzhou-Hengyang(GZ-HY) & 0.11809 \\
9 & Hangzhou(HZ) & 0.07678 & Xian-Zhengzhou(XA-ZZ) & 0.11575 \\
10 & Shenzhen(SZ) & 0.07214 & Tianjin-Jinan(TJ-JN) & 0.11132 \\
\hline
\end{tabular}

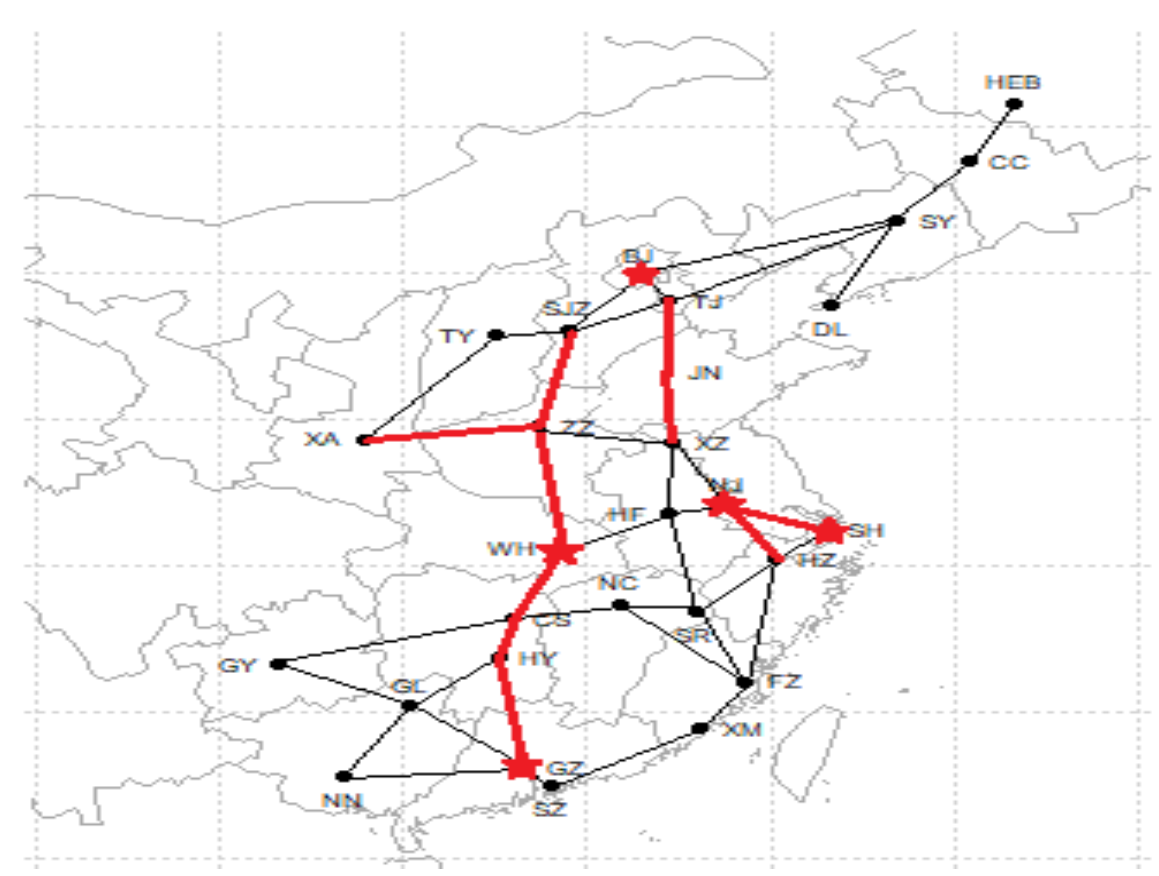

Figure 2. Simplified CRH network map with critical 5 nodes and 10 links highlighted

\section{Simulation analysis of SLFs using Monte Carlo}

As one of the most rapidly growing critical infrastructures in China, the CRH system plays an important role in ensuring economic development and social stability. However, at present, the system faces various types of disruption, including natural disasters, malicious attacks, and random disruptions. Such disruptions are generally highly uncertain events that are difficult to predict, estimate and model, thereby posing significant threats to the continuity of the system's operation. In Section 3, we conducted a train flow-based vulnerability analysis with respect to each individual component in the CRH network. Although the assumption of disruption on a specific component can simplify the calculations, it might not be sufficiently representative when considering certain types of disruptions that affect a whole area of the system. In these cases, the analysis of SLFs is necessary to fully understand the risk and vulnerability of the CRH network.

In reality, geological and hydrological disasters are two serious natural disasters that 
frequently disturb the CRH network. These two disasters are mainly considered here as disruption scenarios and the localized area is determined in terms of provinces. We consider 21 provinces including all the chosen stations in this study. Based on the data obtained from China Statistical Yearbook ${ }^{29}$, we consider the geological and hydrological disasters occurred in these provinces during the period from 2005 to 2015. We obtain the natural disaster probability $n d p \in[0,1]$ based on the statistical mean value for each province per year. As shown in Figure 3, a darker area implies more frequent geological and hydrological disasters occurred. The most serious area for disasters is Hunan Province (including Changsha and Hengyang Stations), which is subject to frequent hydrological and geological disasters. Statistics ${ }^{29}$ indicate that 2,140 geological disasters occurred in Hunan Province in 2015, which caused direct economic losses as much as 878 million RMB. The areas suffering less serious natural disasters are those around Hunan Province, as shown in bright color. Some areas with lighter color are located in the plains or have small precipitation.

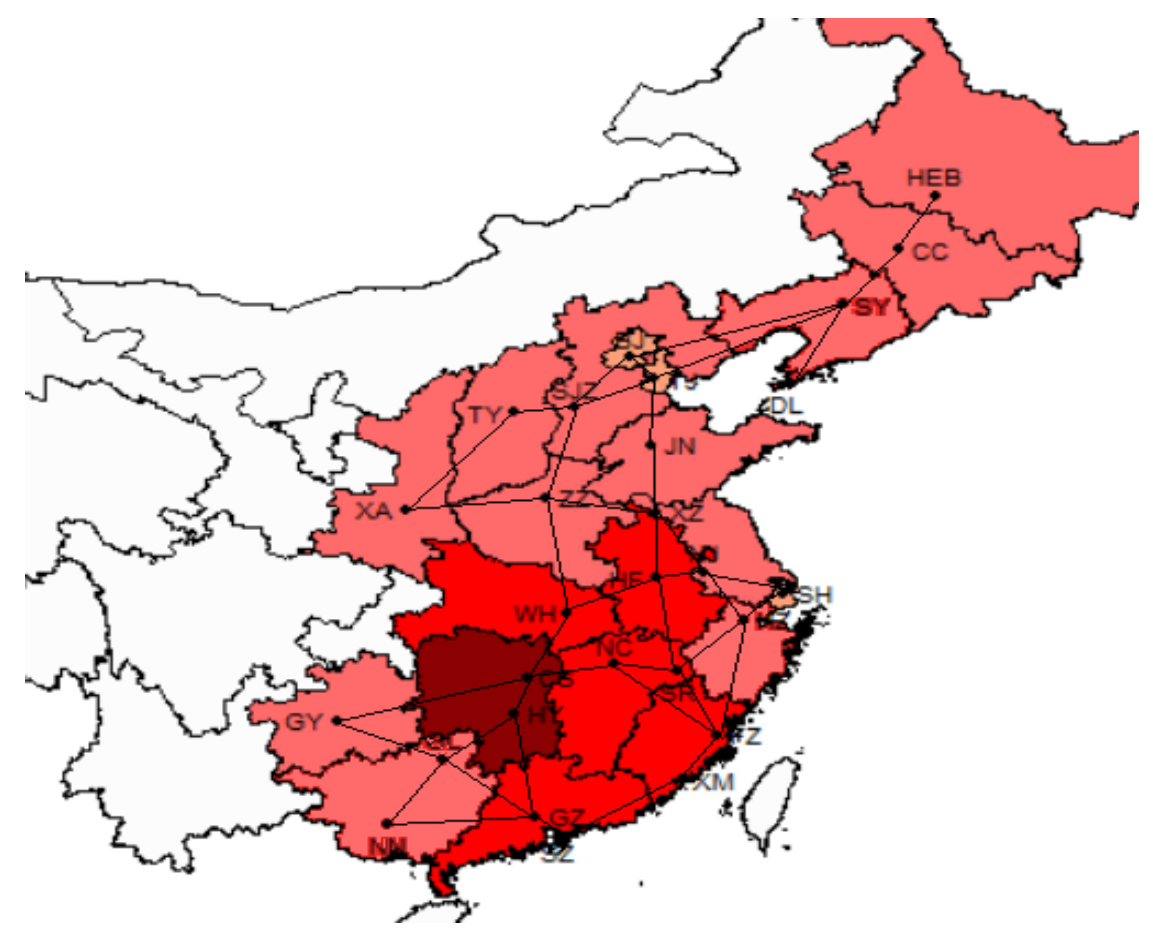

Figure 3. Area distribution of geological and hydrological disasters in China

Most of the disruptions are region-based. For example, typhoon usually rages through the south and southeast, whereas earthquakes often occur in the southwest and floods mostly sweep the central regions along Yangtze River, respectively. We conduct an analysis of SLFs using Monte Carlo simulation, which is a practical tool for studying initiating events in the CRH network, because the components failures are often distributed in a spatially localized area ${ }^{40}$. A set of scenarios $S$ is generated as of the disruptions in the network. Each scenario in $S$ represents a "disruption plan", which can be due to random failures, malicious attacks, natural disasters, or other disruptions. The scenarios set $S$ comprises binary-valued variables 
$x_{s}$ equal to 0 or 1 , where $x_{i s}=0$ indicates that component $i$ is failed in scenario $s$ and $x_{i s}=$ 1 indicates otherwise.

$$
x_{s}=\left\{x_{1 s}, x_{2 s}, \ldots, x_{n s}, x_{(n+1) s}, \ldots, x_{(n+m) s}\right\}
$$

More than one disruption can simultaneously occur in the CRH network during operation. Therefore, some scenarios can actually be superposed. Here, we consider a common disruption scenario that involves the superposition of attacks and natural disasters. Components in the network face a series of initiating events, including various incidents. The specific calculation process is as shown in the flowchart of Figure 4:

Step 1: Define the number of iterations to be simulated; introduce a counter $s$ and set $s=1$.

Step 2: Determine the provinces based on the 28 stations. From the China Statistical Yearbook from 2005 to 2015, determine the natural disaster probabilities for the 21 provinces and define its corresponding stations' natural disaster probability denoted by $n d p_{i}$.

Step 3: In the studied component set, randomly select $0 \%--10 \%$ of them and assume that as a consequence of the disruption they cannot function. Correspondingly, $p_{i}^{S}=1$.

Step 4: For each $n d p_{i}$, generate a uniformly distributed random number $\varepsilon_{i}$ within $[0,1]$, and compare $n d p_{i}$ and $\varepsilon_{i}$ : when $\varepsilon_{i}$ is no larger than $n d p_{i}$, the province is considered to suffer a natural disaster and the stations inside the provinces fail, $p_{i}^{S}=1$; otherwise, $p_{i}^{S}=0$.

Step 5: Update iteration counter $s$. If $s<10000$, then $s=s+1$. Return to Step 3; otherwise, the calculation is finished. Output the mean value of $p_{i}^{s}$ (each node's disruption probability).

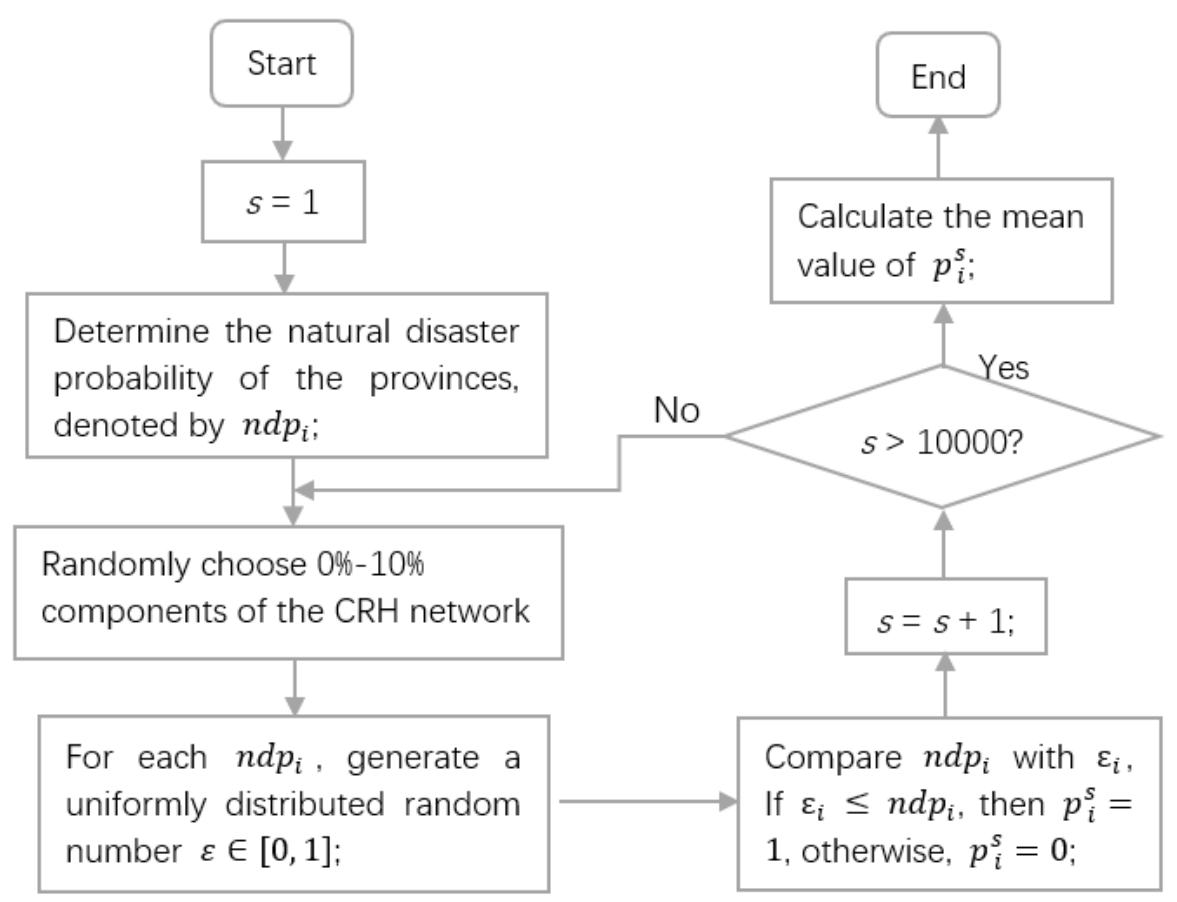

Figure 4. Flowchart of the Monte Carlo simulation under SLF 
After the 10,000 -scenarios simulation, we can obtain the mean value of $p_{i}^{s}$ for each node $i$, which gives the failure frequency of each station node. Taking risk as the product of probability and severity of adverse effects ${ }^{41-44}$, we can calculate the risk of each component. The risk consequence/severity is calculated by the sum of the affected train mileages as discussed in Section 3. The results are shown in Figure 5, in which the areas are filled with varying degrees of color: a darker shade implies greater vulnerability to the high-speed rail operations in that area. The scale value indicates the mileage sum of the CRH affected in that region, in units of $100 \mathrm{~km}$. Given the larger disaster probability, the Hunan Province remains most vulnerable. Another area of equal degree of vulnerability is Guangdong Province, which is also one of the most prosperous areas. As shown in Table 3, within Guangdong Province, Guangzhou and Shenzhen Stations rank both high in terms of flow-based vulnerability. Besides, the province itself suffers a relatively high frequency of natural disasters.

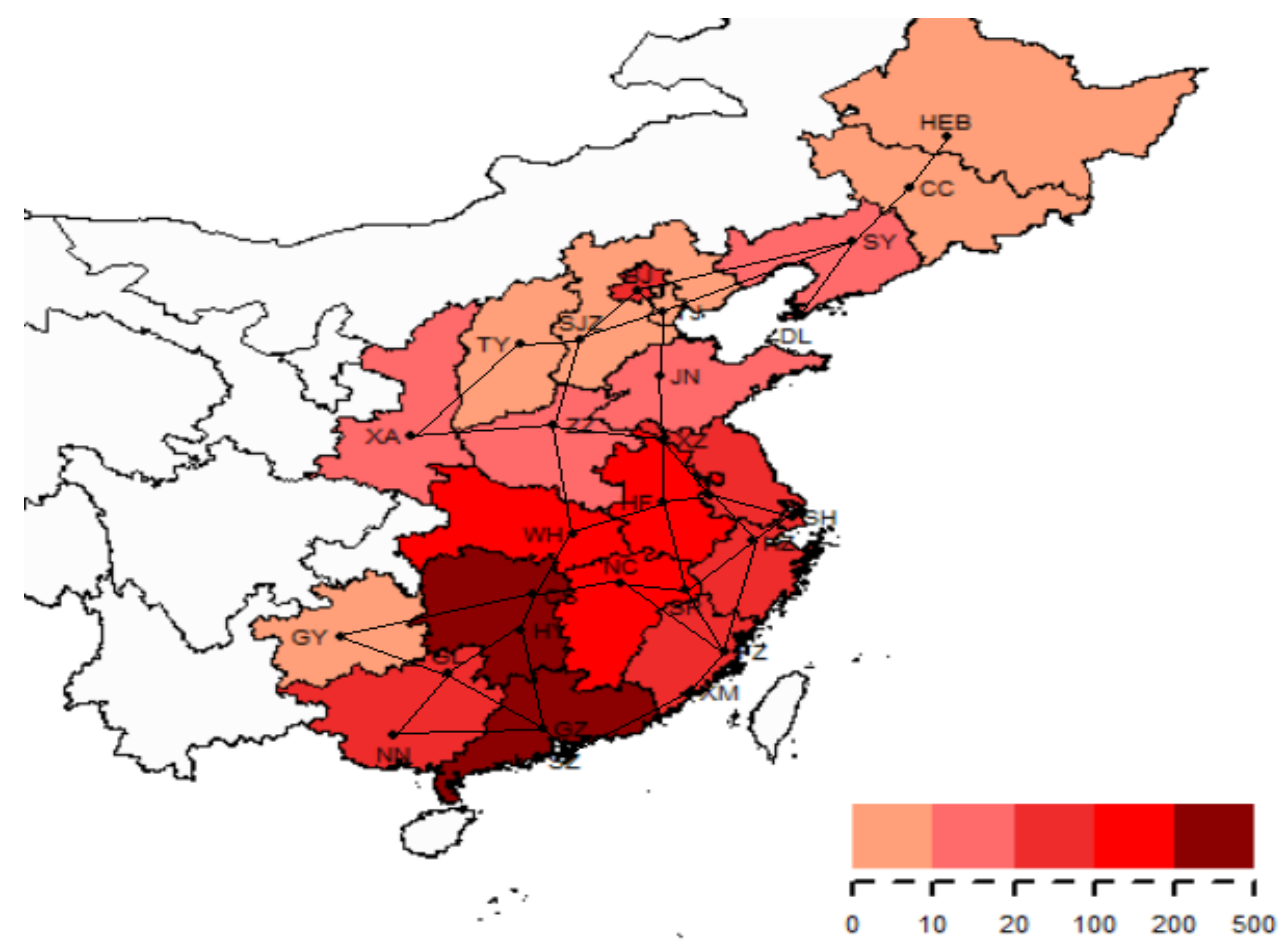

Figure 5. Vulnerability of districts by simulation of SLFs in $100 \mathrm{~km}$ units

\section{Discussions}

The calculation results of the topological and flow-based vulnerability analyses shown some differences. For example, nodes that are critical for the flow-based vulnerability analysis may not be important in the topological analysis. We take Beijing for a comprehensive example. From Section 2.2, the betweenness of Beijing is only 0.07047, ranking 25 th among the 28 stations, which can be explained by the city's geographic position. However, in reality, Beijing is an important hub, because of the population, economy and also political positions. With the consideration of train mileage, the importance of this station 
increases greatly and ranks 2 nd among the 28 stations. Given that many trains start or end at Beijing Station, if a disruption occurs, these service lines would be affected considerably. With the consideration of train mileage, Shanghai is the most vulnerable station. However, similar to Beijing Station, this node does not stand out when analyzed from a topological or network efficiency perspective. The same difference can also be found in the importance comparison of links in terms of vulnerability. The most vulnerable link in terms of train flow is Shanghai-Nanjing. However, this link is not important in the topological analysis (only ranks 35th among all 42 links). This phenomenon can be explained by the different meaning of the three metrics: the betweenness centrality emphasizes the components' position and role as a hub in the network topology; the connectivity efficiency looks at the entire network for efficiently transmitting among the nodes and re-routing; the flow-based vulnerability analysis investigates the effect of each component's failure on the operating travel mileages.

With the consideration of spatially localized failures, and corresponding consequences, we analyze the vulnerability distribution on the CRH map, which considers the importance of each district from a comprehensive perspective. The areas with high vulnerability should be prepared and emergency responses are required to recover from the impact. The results of the analysis also provide insights for the investments of planning and maintenance of the CRH system. For example, in the southeast provinces, train stations must be designed to withstand the impact of typhoons; railroad tracks in flood-prone areas may need to be laid on higher terrains or viaducts; railroad tracks in the frozen areas need to be designed for frost protection. Moreover, decision-makers can rely on the flow-based vulnerability analysis to plan contingency management, such as temporary scheduling and evacuation of passengers.

For the failure consequences shown in Section 3, nodes such as Shanghai and Beijing, indicate a high flow-based vulnerability because many train service lines start or end there. In the event of an interruption, these service lines are disrupted and, thus, seriously affect public safety, economic development and social stability. Managers often consider investment to reinforce the stations and increase train services in these important but already overburdened stations, while not realizing that it may result in high vulnerability. In terms of diversion, constructing two or more stations in prosperous and vulnerable cities is becoming common and convenient to reduce the risk of the entire $\mathrm{CRH}$ network. The failure probability of a train service line will disperse, that is, even when one of the stations breakdowns, the others can continue to operate as a substitute. Although many cities now have multiple stations, most of them are not adequate for the operation of high-speed trains. As shown in Figure 6, we select five nodes with multiple stations and calculate their train flow-based vulnerability separately. The comparison indicates that the vulnerability of different stations in one city varies significantly. This is mainly due to the uneven distribution of high-speed train services for various reasons. For example, in Section 3 we found that Shanghai is an important site whose 
failure can seriously affect the operation of CRH. Calculating the vulnerability of each station in Shanghai separately, Shanghai Hongqiao Station accounts for a large proportion of more than $87 \%$ in terms of disturbed cumulative train mileage. Other than scattering the high-speed passenger train services and balancing the vulnerability, a solution of constructing a comprehensive hub is adopted in Shanghai, called Hongqiao Transportation Hub. Hongqiao integrated transport hub is an innovation in urban transport construction, which includes a variety of modes of transport such as aviation, high-speed railway, maglev, freeway and subway combined together. In this manner, passengers can be quickly evacuated to another system both for transit service, and for facing disruptions or emergencies. For example, travelers can decide to take airplanes when the high-speed rail services are unavailable in the same hub. Furthermore, the vulnerability of interdependent transportation networks will be more complicated and involve more factors.

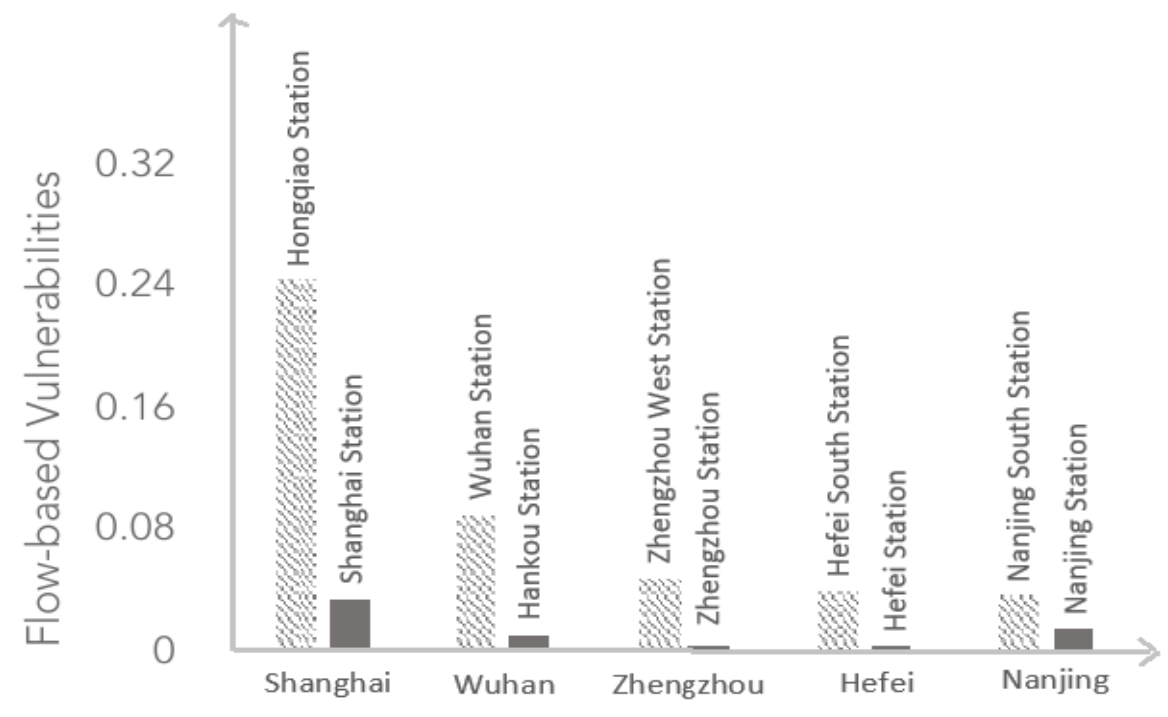

Figure 6. Comparison of the flow-based vulnerability in separated stations

Combined disruptions sometimes occur in the high-speed rail, which cause more than one component failed in the network simultaneously. Table 4 shows the calculation results under pair-disturbance scenarios. The first three vulnerable combinations in Situation 1 are Beijing with Shanghai, Guangzhou with Shanghai, and Wuhan with Shanghai. All these nodes are also most vulnerable separately, as shown in Table 3 and Figure 2. As Shanghai is the most flow-based vulnerable site, 9 out of top-ten pairs include Shanghai. The combinations in Situations 2 and 3 indicate consistent results, showing that components alone are also important when combined with others, such as in Shanghai Station and links of Wuhan--Changsha and Shanghai--Nanjing. It is noted that once these vulnerable components fail simultaneously, the entire network functioning may fall into chaos, and require significant resources and time to recover.

TABLE 4. TRAIN FLOW-BASED VULNERABILITY OF CRH UNDER PAIR-DISTURBANCE SCENARIOS 


\begin{tabular}{|c|c|c|c|c|c|c|}
\hline $\begin{array}{c}\text { Ranki } \\
\text { ng }\end{array}$ & $\begin{array}{l}\text { Nodes } \\
+ \text { Nodes }\end{array}$ & $\mathbf{V}^{F}$ & $\begin{array}{l}\text { Nodes } \\
\text { + Links }\end{array}$ & $\mathbf{V}^{F}$ & $\begin{array}{c}\text { Links } \\
+ \text { Links }\end{array}$ & $\mathbf{V}^{F}$ \\
\hline 1 & $\mathrm{BJ}+\mathrm{SH}$ & 0.46489 & $\mathrm{SH}+\mathrm{WH}-\mathrm{CS}$ & 0.46768 & SH-NJ + WH-CS & 0.42184 \\
\hline 2 & $\mathrm{GZ}+\mathrm{SH}$ & 0.41064 & $\mathrm{SH}+\mathrm{WH}-\mathrm{ZZ}$ & 0.45004 & SH-NJ + WH-ZZ & 0.40419 \\
\hline 3 & $\mathrm{WH}+\mathrm{SH}$ & 0.40519 & $\mathrm{SH}+\mathrm{CS}-\mathrm{HY}$ & 0.42101 & $\mathrm{SH}-\mathrm{NJ}+\mathrm{CS}-\mathrm{HY}$ & 0.38121 \\
\hline 4 & $\mathrm{CS}+\mathrm{SH}$ & 0.37887 & $\mathrm{SH}+\mathrm{SJZ}-\mathrm{ZZ}$ & 0.41766 & SH-NJ + SJZ-ZZ & 0.37182 \\
\hline 5 & $\mathrm{XA}+\mathrm{SH}$ & 0.36669 & $\mathrm{BJ}+\mathrm{SH}-\mathrm{NJ}$ & 0.41642 & BJ-NJ + GZ-HY & 0.35298 \\
\hline 6 & $\mathrm{BJ}+\mathrm{GZ}$ & 0.36381 & $\mathrm{SH}+\mathrm{BJ}-\mathrm{HZ}$ & 0.40845 & $\mathrm{WH}-\mathrm{CS}+\mathrm{JN}-\mathrm{XZ}$ & 0.34416 \\
\hline 7 & $\mathrm{ZZ}+\mathrm{SH}$ & 0.36365 & $\mathrm{SH}+\mathrm{GZ}-\mathrm{HY}$ & 0.39511 & SH-NJ + SJZ-BJ & 0.34001 \\
\hline 8 & $\mathrm{HZ}+\mathrm{SH}$ & 0.36128 & $\mathrm{SH}+\mathrm{SJZ}-\mathrm{BJ}$ & 0.38585 & SH-NJ + NJ-HZ & 0.33335 \\
\hline 9 & $\mathrm{NJ}+\mathrm{SH}$ & 0.35725 & $\mathrm{BJ}+\mathrm{WH}-\mathrm{CS}$ & 0.38449 & SH-NJ + SR-NC & 0.33204 \\
\hline 10 & $\mathrm{SZ}+\mathrm{SH}$ & 0.35287 & $\mathrm{SH}+\mathrm{JN}-\mathrm{XZ}$ & 0.37897 & $\mathrm{SH}-\mathrm{NJ}+\mathrm{JN}-\mathrm{XZ}$ & 0.32651 \\
\hline
\end{tabular}

\section{Conclusion}

In this study, we propose a comprehensive vulnerability analysis of $\mathrm{CRH}$, which includes topological and train flow-based vulnerability analysis. Given the different importance of each train service line, the vulnerability defined here is the sum of affected train mileage, rather than the number of affected trains as done in traditional research. An SLF-induced vulnerability simulation is conducted using the Monte Carlo method. First-tier cities, such as Beijing, Shanghai, and Guangzhou, are vulnerable under disruptions due to their prosperous population and large number of high-speed passenger trains that depart from/arrive at the stations. Once they are interrupted, the service lines that include these stations will be affected considerably, thereby causing unexpected losses. Therefore, strengthening of these vital stations should be given attention. Moreover, dividing these start/end sites is feasible, such that the probability of failure in this line can decrease considerably. That is, if we construct two or more high-speed rail stations in these important cities, even if one of the stations breaks down, the others can continue to operate as a substitute. Another emerging solution of constructing transportation hubs integrating high-speed rail, aviation and other transportation systems is currently developed in various cities, after the success of Shanghai Hongqiao hub. From a managerial perspective regarding vulnerability, the traffic flow of nodes in the middle of service lines can be increased by enabling some trains to start from or end at these second- or third-tier cities. The current trend tends to increase the number of vehicles on stations that are already highly important and busy. However, an optimal balance should be reached between the operational gains and vulnerability mitigation. As $\mathrm{CRH}$ has an obvious regional disaster characteristic according to the risk distribution map, specific defensive efforts and contingency response should be designed against disruptions. 
In the future work, uncertainty propagation and control regarding vulnerability will be studied taking into account restoration and timetable rescheduling of trains under disruptions. The proposed approach can also be extended to other critical transport infrastructures. Furthermore, the cascading and complementary effects of interdependent transportation networks under disruptions will be considered.

\section{Reference}

1. Murray AT and Grubesic TH. Critical infrastructure protection: The vulnerability conundrum. Telematics and Informatics. 2012; 29: 56-65.

2. Park J, Seager TP, Rao PS, Convertino M and Linkov I. Integrating risk and resilience approaches to catastrophe management in engineering systems. Risk Analysis. 2013; 33: 356.

3. Pursiainen C. The Challenges for European Critical Infrastructure Protection. Journal of European Integration. 2009; 31: 721-39.

4. Yusta JM, Correa GJ and Lacal-Arántegui R. Methodologies and applications for critical infrastructure protection: State-of-the-art. Energy Policy. 2011; 39: 6100-19.

5. Marrone S, Nardone R, Tedesco A, et al. Vulnerability modeling and analysis for critical infrastructure protection applications. International Journal of Critical Infrastructure Protection. 2013; 6: 217-27.

6. Fang YP, Pedroni N and Zio E. Resilience-Based Component Importance Measures for Critical Infrastructure Network Systems. IEEE Transactions on Reliability. 2016; 65: 502-12.

7. Zio E. Challenges in the vulnerability and risk analysis of critical infrastructures. Reliability Engineering \& System Safety. 2016; 152: 137-50.

8. Johansson J, Hassel $\mathrm{H}$ and Zio E. Reliability and vulnerability analyses of critical infrastructures: Comparing two approaches in the context of power systems. Reliability Engineering \& System Safety. 2013; 120: 27-38.

9. Ouyang M. Review on modeling and simulation of interdependent critical infrastructure systems. Reliability Engineering \& System Safety. 2014; 121: 43-60.

10. Berdica K. An introduction to road vulnerability: what has been done, is done and should be done. Transport Policy. 2002; 9: 117-27.

11. Jenelius E, Petersen $T$ and Mattsson LG. Importance and exposure in road network vulnerability analysis. Transportation Research Part A Policy \& Practice. 2006; 40: 537-60.

12. Jenelius E. Network structure and travel patterns: explaining the geographical disparities of road network vulnerability. Journal of Transport Geography. 2009; 17: 234-44.

13. El-Rashidy RA and Grant-Muller SM. An assessment method for highway network vulnerability. Journal of Transport Geography. 2014; 34: 34-43.

14. Li W and Cai X. Empirical analysis of a scale-free railway network in China. Physica A: Statistical Mechanics and its Applications. 2007; 382: 693-703.

15. Johansson J and Hassel $\mathrm{H}$. An approach for modelling interdependent infrastructures in the context of vulnerability analysis. Reliability Engineering \& System Safety. 2010; 95: 1335-44.

16. Ouyang M, Pan ZZ, Hong $L$ and He Y. Vulnerability analysis of complementary transportation systems with applications to railway and airline systems in China. Reliability Engineering \& System Safety. 2015; 142: 248-57. 
17. Bocchini P, Frangopol, D. M., Ummenhofer, T., Zinke, T. Resilience and sustainability of civil infrastructure: Toward a unified approach. Journal of Infrastructure Systems. 2014; 20: 1-16.

18. Chang SE and Nojima N. Measuring post-disaster transportation system performance: the 1995 Kobe earthquake in comparative perspective. Transportation research part A: policy and practice. 2001; 35: 475-94.

19. Lee II EE, Mitchell JE and Wallace WA. Restoration of services in interdependent infrastructure systems: A network flows approach. IEEE Transactions on Systems, Man, and Cybernetics, Part C (Applications and Reviews). 2007; 37: 1303-17.

20. Nurre SG, Cavdaroglu B, Mitchell JE, Sharkey TC and Wallace WA. Restoring infrastructure systems: An integrated network design and scheduling (INDS) problem. European Journal of Operational Research. 2012; 223: 794-806.

21. Cavdaroglu B, Hammel E, Mitchell JE, Sharkey TC and Wallace WA. Integrating restoration and scheduling decisions for disrupted interdependent infrastructure systems. Annals of Operations Research. 2013; 203: 279-94.

22. Zhu $Y$ and Goverde RM. Railway timetable rescheduling with flexible stopping and flexible short-turning during disruptions. Transportation Research Part B: Methodological. 2019; 123: 149-81.

23. Lee li EE, Mitchell JE and Wallace WA. Restoration of Services in Interdependent Infrastructure Systems: A Network Flows Approach. IEEE Transactions on Systems, Man and Cybernetics, Part C (Applications and Reviews). 2007; 37: 1303-17.

24. Jenelius $E$ and Mattsson L-G. Road network vulnerability analysis of area-covering disruptions: A grid-based approach with case study. Transportation Research Part A: Policy and Practice. 2012; 46: 746-60.

25. Wang $S$, Hong $L$ and Chen $X$. Vulnerability analysis of interdependent infrastructure systems: A methodological framework. Physica A: Statistical Mechanics and its Applications. 2012; 391: 3323-35.

26. Nicholson $\mathrm{CD}$, Barker $\mathrm{K}$ and Ramirez-Marquez JE. Flow-based vulnerability measures for network component importance: Experimentation with preparedness planning. Reliability Engineering \& System Safety. 2016; 145: 62-73.

27. Chen X. A Sustainability Analysis on the Wuhan-Guangzhou High-Speed Railway in China. International Journal of Sustainable Transportation. 2014; 9: 348-63.

28. Banister D and Givoni M. High-Speed Rail in the EU27: Trends, Time, Accessibility and Principles. Built Environment. 2013; 39: 324-38(15).

29. "Yearbook". China's yearbook on transportation and communications. Year Book House of China Transportation \& Communications, Beijing. 2017.

30. Cao J, Liu XC, Wang Y and Li Q. Accessibility impacts of China's high-speed rail network. Journal of Transport Geography. 2013; 28: 12-21.

31. Jiao J, Wang J, Jin F and Dunford M. Impacts on accessibility of China's present and future HSR network. Journal of Transport Geography. 2014; 40: 123-32.

32. Rongfang L. The Role of State: High Speed Rail Development in China. Advances in Management. 2015; 8.

33. Ouyang M. Critical location identification and vulnerability analysis of interdependent infrastructure systems under spatially localized attacks. Reliability Engineering \& System Safety. 2016; 154: 106-16. 
34. Ouyang M. A Mathematical Framework to Optimize Resilience of Interdependent Critical Infrastructure Systems under Spatially Localized Attacks. European Journal of Operational Research. 2017; 262: 1072-84.

35. GAOTIE.CN. High-speed rail network. 2017.

36. Macchia $M$, Centrone D, Fumagalli $L$ and Pavirani GP. Maintenance management of railway infrastructures based on reliability analysis. Reliability Engineering \& System Safety. 2012; 104: 71-83.

37. Peeta S, Salman FS, Gunnec D and Viswanath K. Pre-disaster investment decisions for strengthening a highway network. Computers \& Operations Research. 2010; 37: 1708-19.

38. Fang $\mathrm{Y}$, Pedroni $\mathrm{N}$ and Zio E. Optimization of Cascade-Resilient Electrical Infrastructures and its Validation by Power Flow Modeling. Risk Analysis An Official Publication of the Society for Risk Analysis. 2015; 35: 594.

39. Fang YP, Pedroni N and Zio E. Comparing Network-Centric and Power Flow Models for the Optimal Allocation of Link Capacities in a Cascade-Resilient Power Transmission Network. IEEE Systems Journal. 2017; PP: 1-12.

40. Ouyang $M$, Tian $H$, Wang $Z$, Hong $L$ and Mao Z. Critical infrastructure vulnerability to spatially localized failures with applications to Chinese railway system. Risk Analysis. 2019; 39: 180-94.

41. Aven T. On some recent definitions and analysis frameworks for risk, vulnerability, and resilience. Risk Analysis. 2011; 31: 515-22.

42. Aven T. Risk assessment and risk management: Review of recent advances on their foundation. European Journal of Operational Research. 2016; 253: 1-13.

43. Aven T and Zio E. Quality of Risk Assessment: Definition and Verification. 2017.

44. Flage R, Aven T, Zio E and Baraldi P. Concerns, Challenges, and Directions of Development for the Issue of Representing Uncertainty in Risk Assessment. Risk Analysis. 2014; 34: 1196-207. 